\title{
Melanin Pigmentation of Oral Mucosa in Bangladeshi Population, with Special Reference to Tobacco Habits
}

\author{
Anwar Yousuf ${ }^{1}$, Kei Sakamoto $^{1}$, Rahim Ullah Choudhury ${ }^{2}$ and Akira Yamaguchi ${ }^{1}$ \\ ${ }^{1}$ Oral Pathology, Department of Oral Restitution, Graduate School, Tokyo Medical \& Dental University, Tokyo, Japan. \\ ${ }^{2}$ Department of Oral \& Maxillofacial Surgery, Dhaka Dental College \& Hospital, Dhaka, Bangladesh.
}

\begin{abstract}
Yousuf A, Sakamoto K, Choudhury RU and Yamaguchi A. Melanin pigmentation of oral mucosa in Bangladeshi population, with special reference to tobacco habits. Oral Med Pathol 2005; 10: 57-61, ISSN 1342-0984
\end{abstract}

\begin{abstract}
Abnormal melanin pigmentation of the oral mucosa has been linked to smoking and has been designated smoker melanosis. Since the incidence of smoker melanosis varies among races, we investigated the incidence of melanin pigmentation in 280 Bangladeshi individuals. The incidence of melanin pigmentation was $\mathbf{8 2 . 0 \%}$ in the smoker group and $58.3 \%$ in the non-smoker group. Melanin pigmentation frequently occurred in participants aged from 20-49 years old in the smoker group, while it occurred in participants aged from 30-59 years old in the nonsmoker group. Gingiva and buccal mucosa were frequently affected sites in both the smoker and non-smoker groups. The smoker group had tendency to show more intense melanin pigmentation than that in the non-smoker group. Since the present study is the first extensive survey of smoker melanosis in Bangladesh, these results provide important information for investigating the effects of smoking habits on melanin pigmentation of the oral mucosa among various ethnic groups.
\end{abstract}

Key Words: Melanin pigmentation, Oral mucosa, Smoking, Bangladesh

Correspondence: Anwar Yousuf, Oral Pathology, Department of Oral Restitution, Graduate School, Tokyo Medical \& Dental University, 1-5-45 Yushima, Bunkyo-ku, Tokyo 113-8549, Japan Phone: +81-3-5803-5454, Fax: +81-3-5803-0188, E-mail: aryousuf@hotmail.com

\section{Introduction}

The color of oral mucosa is determined by the degree of keratinization, the thickness of epithelium, the vascular supply and the melanin pigment. Melanin is the commonest endogenous pigment contributing to the color of oral mucosa. Specialized pigmented cells, called melanocytes and situated in the basal layer of the oral epithelium and epidermis, produce melanin. Melanin synthesized in melanocytes is transferred into the cytoplasm of adjacent keratinocytes by the dendritic processes of melanocytes. Melanin can also be found in macrophages, called melanophages, in the lamina propria of oral mucosa, which have ingested melanin granules extruded from melanocytes. Thus, the number and activity of melanocytes and the accumulated level of melanin in keratinocytes and melanophages critically determine the color due to melanin pigmentation.

Melanin pigmentation of oral mucosa occurs under various conditions. Systemic conditions, such as
Addison's disease, Peutz-Jeghers syndrome, McCuneAlbright syndrome, Laugier-Hunziker syndrome and von Rechlinghausen's disease, are often associated with oral melanin pigmentation (1). Smoking is an important local factor affecting melanin pigmentation in oral mucosa. In 1977, Hedin (2) reported the relation of oral melanosis and smoking habit by extensive clinical study in Swedish population, and he first gave the name "smoker's melanosis" to the melanin pigmentation which occurred in a smoker gingiva. Since his report, various data have been accumulated concerning several races (2-14). Although these results prove the close relationship between oral melanosis and smoking habit, the incidence of smoker melanosis varies among populations examined. Further investigation in various races is required to evaluate the exact incidence and etiology of smoker melanosis in oral mucosa.

In Bangladesh, clinicians have recognized oral melanin pigmentation as a common finding, but no de- 
Table 1: Distribution of patients with or without melanin pigmentation according to smoking habit

\begin{tabular}{l|rc|cc|cc}
\hline \multicolumn{1}{c|}{ Groups } & \multicolumn{2}{|c|}{ Melanin } & \multicolumn{2}{c|}{ Non-melanin } & \multicolumn{3}{c}{ Total } \\
& $\mathrm{n}$ & $(\%)$ & $\mathrm{n}$ & $(\%)$ & $\mathrm{n}$ & $(\%)$ \\
\hline Non-smoker & 21 & $(58.3)$ & 15 & $(41.7)$ & 36 & $(100)$ \\
Smoker & 200 & $(82.0)$ & 44 & $(18.0)$ & 244 & $(100)$ \\
\hline
\end{tabular}

Table 2: Distribution of melanin pigmentation according to gender and age in smoker and non-smoker groups Smoker group

\begin{tabular}{l|rrrr|rr}
\hline Age & \multicolumn{2}{|c}{ Male } & \multicolumn{2}{c|}{ Female } & \multicolumn{2}{c}{ Total } \\
& \multicolumn{1}{|c}{$\mathrm{n}$} & $(\%)$ & $\mathrm{n}$ & $(\%)$ & $\mathrm{n}$ & $(\%)$ \\
\hline $10-19$ & 5 & $(3.3)$ & 1 & $(2.0)$ & 6 & $(3.0)$ \\
$20-29$ & 36 & $(24.0)$ & 21 & $(42.0)$ & 57 & $(28.5)$ \\
$30-39$ & 25 & $(16.7)$ & 28 & $(56.0)$ & 53 & $(26.5)$ \\
$40-49$ & 49 & $(32.7)$ & 0 & $(0.0)$ & 49 & $(24.5)$ \\
$50-59$ & 28 & $(18.7)$ & 0 & $(0.0)$ & 28 & $(14.0)$ \\
$60-69$ & 3 & $(2.0)$ & 0 & $(0.0)$ & 3 & $(1.5)$ \\
$70-79$ & 4 & $(2.6)$ & 0 & $(0.0)$ & 4 & $(2.0)$ \\
\hline Total & 150 & $(100)$ & 50 & $(100)$ & 200 & $(100)$ \\
\hline
\end{tabular}

\begin{tabular}{l|rrrr|rr}
\multicolumn{1}{l}{ Non-smoker group } \\
\hline Age & \multicolumn{2}{|c}{ Male } & \multicolumn{2}{c|}{ Female } & \multicolumn{2}{c}{ Total } \\
& $\mathrm{n}$ & $(\%)$ & $\mathrm{n}$ & $(\%)$ & $\mathrm{n}$ & $(\%)$ \\
\hline $10-19$ & 0 & $(0.0)$ & 0 & $(0.0)$ & 0 & $(0.0)$ \\
$20-29$ & 0 & $(0.0)$ & 0 & $(0.0)$ & 0 & $(0.0)$ \\
$30-39$ & 10 & $(55.5)$ & 3 & $(100.0)$ & 13 & $(61.9)$ \\
$40-49$ & 5 & $(27.8)$ & 0 & $(0.0)$ & 5 & $(23.8)$ \\
$50-59$ & 3 & $(16.7)$ & 0 & $(0.0)$ & 3 & $(14.3)$ \\
$60-69$ & 0 & $(0.0)$ & 0 & $(0.0)$ & 0 & $(0.0)$ \\
$70-79$ & 0 & $(0.0)$ & 0 & $(0.0)$ & 0 & $(0.0)$ \\
\hline Total & 18 & $(100)$ & 3 & $(100)$ & 21 & $(100)$ \\
\hline
\end{tabular}

tails have been compiled and reported. Tobacco habits, which include cigarette smoking, pipe with raw tobacco, and smokeless tobacco in the form of snuff or chewing tobacco, are very popular in Bangladesh. Among these habits, cigarette smoking is the most popular. Thus we investigated the incidence of melanin pigmentation in oral mucosa, with special reference to smoking habits. We here demonstrate that the Bangladeshi population exhibits a higher incidence of melanin pigmentation associated with smoking compared with those data reported from other countries.

\section{Materials and methods}

Two hundred eighty individuals (230 males and 50 females) were selected by a simple random sampling method from the list of participants referred to Dhaka Dental College and Hospital, Department of Oral and Maxillofacial Surgery. Participants who had any systemic complicating factors were excluded from this survey. We first investigated clinically melanin pigmentation in the oral mucosa of these participants. Information on smoking history was collected from all of the participants. The degree of pigmentation was classified according to the scale used by Unsal et al. with a minor modification (12). Degree I: slight melanin pigmentation at one or more localized areas. Degree II: moderate melanin pigmentation that converges to in short continuous ribbons. Degree III: heavy melanin pigmentation with extensive areas that converge into long continuous ribbons.

To confirm the presence of melanin pigment histologically, we performed biopsy from 50 participants randomly chosen from the 280 participants. Biopsy specimens were embedded in paraffin after fixation with $10 \%$ formalin, and sections of $4 \mu \mathrm{m}$ thickness were prepared. They were stained with hematoxylin and eosin for studying the general histopathological findings. Melanin was 
Table 3: Sites of melanin pigmentation in smoker and non-smoker groups Smoker group

\begin{tabular}{lrr}
\hline \multicolumn{1}{c}{ Site } & $\mathrm{n}$ & $(\%)$ \\
\hline Lip & 25 & $(12.5)$ \\
Gingiva & 105 & $(52.5)$ \\
Buccal-mucosa & 50 & $(25.0)$ \\
Tongue & 5 & $(2.5)$ \\
Palate & 15 & $(7.5)$ \\
\hline Total & 200 & $(100)$ \\
\hline Non-smoker group & & \\
\hline \multicolumn{1}{c}{ Site } & $\mathrm{n}$ & $(\%)$ \\
\hline Lip & 0 & $(0.0)$ \\
Gingiva & 18 & $(85.7)$ \\
Buccal-mucosa & 3 & $(14.3)$ \\
Tongue & 0 & $(0.0)$ \\
Palate & 0 & $(0.0)$ \\
\hline Total & 21 & $(100)$ \\
\hline
\end{tabular}

visualized by staining with the Masson-Fontana silver reduction method. To exclude hemosiderin or exogenous pigmentation such as metal particles, the sections were submitted to bleaching. The presence of melanin was evaluated as follows. Mild: slight melanin pigmentation was observed in a localized area. Moderate: extensive melanin pigmentation was observed in a scattered fashion. Severe: melanin pigmentation showed extensive, and heavy density was continuously observed in the basal layer.

\section{Results}

\section{Clinical features of melanin pigmentation}

Clinical observation of 280 participants revealed that $221(78.9 \%)$ showed melanin pigmentation of oral mucosa and $59(21.1 \%)$ exhibited no apparent pigmentation. Out of 280 participants, 244 (87.1\%) were smoker and $36(12.9 \%)$ were non-smoker. The incidence of melanin pigmentation in the smoker group [200 out of 244 $(82.0 \%)]$ was higher than that of the non-smoker group [21 out of $36(58.3 \%)$ ] (Table 1).

Table 2 summarizes distribution of 221 participants who exhibited melanin pigmentation in both groups of smoker and non-smoker, according to age and gender. In general, the melanin pigmentation occurred with a higher frequency in the $3^{\text {rd }}, 4^{\text {th }}$ and $5^{\text {th }}$ decades than in other decades in the smoker group, whereas the pigmentation was observed in the $4^{\text {th }}, 5^{\text {th }}$ and $6^{\text {th }}$ decades, but not in the $3^{\text {rd }}$ decade in non-smoker group.

In males, melanin pigmentation occured in all decades in the smoker group, but it occurred only in the $3^{\text {rd }}$, $4^{\text {th }}$ and $5^{\text {th }}$ decades in the non-smoker group. In the male smoker group, the $5^{\text {th }}$ decade showed the highest frequency followed by the $3^{\text {rd }}, 6^{\text {th }}$ and $4^{\text {th }}$ decades. In female participants, melanin pigmentation was frequently ob- served in smoker in the $3^{\text {rd }}$ and $4^{\text {th }}$ decades.

Table 3 summarizes the sites of melanin pigmentation in the oral cavity. Gingiva was the most frequently affected site in both the smoker and non-smoker groups. Buccal mucosa was also a favorite site in both groups. In the smoker group, melanin pigmentation also occurred in the lip, tongue and palate, but no pigmentation was observed in these sites in the non- smoker group. There were no cases that exhibited melanin pigmentation at multiple sites in the oral cavity. Table 4 demonstrates intensity of melanin pigmentation in the smoker and nonsmoker groups. Among 200 smoker participants, 130 (65.0\%) were classified as Grade I, 37 (18.5\%) were Grade II, and 33 (16.5\%) were Grade III. In non-smoker group, 17 out of 21 participants ( $81.0 \%$ ) were Grade I and 4 out of 21 participants (19.0\%) were Grade II. There were no participants who exhibited Grade III melanosis among the non-smoking participants. Table 4 also shows intensity of melanin pigmentation according to the affected sites. In the smoking group, Grade III melanin pigmentation occurred in the lip, gingiva and buccal mucosa, but no cases of Grade III appeared in the tongue and palate. In the non-smoker group, melanin pigmentation was observed in only the gingiva and buccal mucosa, and the intensity of the pigmentation in all of the cases belonged to Grade I or Grade II.

\section{Histological findings}

Histological investigation of 50 participants revealed that 8 cases diagnosed as melanin pigmentation by clinical examination had intense exogenous metal pigmentation associated with a limited amount of melanin. These cases were classified as mild by histological evaluation. Other cases exhibited extensive melanin pigmentation and they were classified as moderate ( 15 cases) or 
Table 4: Distribution of melanin pigmentation according to intensity and sites in smoker and non-smoker groups Smoker group

\begin{tabular}{|c|c|c|c|c|c|c|c|c|c|c|}
\hline \multirow[t]{2}{*}{ Degree } & \multicolumn{2}{|r|}{ Lip } & Gingiva & \multicolumn{2}{|c|}{$\begin{array}{l}\text { Buccal } \\
\text { mucosa }\end{array}$} & \multicolumn{2}{|c|}{ Tongue } & \multicolumn{2}{|c|}{ Palate } & Total \\
\hline & $\mathrm{n}$ & $(\%)$ & $(\%)$ & $\mathrm{n}$ & & $\mathrm{n}$ & $(\%)$ & $\mathrm{n}$ & $(\%)$ & $(\%)$ \\
\hline Grade-I & 10 & $(40.0)$ & $65(62.0)$ & 42 & $(84.0)$ & 3 & $(60.0)$ & 10 & $(66.7)$ & $130(65.0)$ \\
\hline Grade-II & 6 & $(24.0)$ & $20(19.0)$ & 4 & $(8.0)$ & 2 & $(40.0)$ & 5 & $(33.3)$ & $37(18.5)$ \\
\hline Grade-III & 9 & $(36.0)$ & $20(19.0)$ & 4 & $(8.0)$ & 0 & $(0.0)$ & 0 & $(0.0)$ & $33(16.5)$ \\
\hline Total & 25 & $(100)$ & $105(100)$ & 50 & (100) & 5 & $(100)$ & 15 & $(100)$ & $200(100)$ \\
\hline
\end{tabular}

Non-smoker group

\begin{tabular}{l|rr|rr|rr|rr|rr|rr}
\hline Degree & \multicolumn{2}{|c|}{ Lip } & \multicolumn{2}{|c|}{ Gingiva } & \multicolumn{2}{c|}{$\begin{array}{c}\text { Buccal } \\
\text { mucosa }\end{array}$} & \multicolumn{2}{|c|}{ Tongue } & \multicolumn{2}{|c|}{ Palate } & \multicolumn{2}{|c}{ Total } \\
& $\mathrm{n}$ & $(\%)$ & $\mathrm{n}$ & $(\%)$ & $\mathrm{n}$ & $(\%)$ & $\mathrm{n}$ & $(\%)$ & $\mathrm{n}$ & $(\%)$ & $\mathrm{n}$ & $(\%)$ \\
\hline Grade-I & 0 & $(0.0)$ & 15 & $(83.3)$ & 2 & $(66.7)$ & 0 & $(0.0)$ & 0 & $(0.0)$ & 17 & $(81.0)$ \\
Grade-II & 0 & $(0.0)$ & 3 & $(16.7)$ & 1 & $(33.3)$ & 0 & $(0.0)$ & 0 & $(0.0)$ & 4 & $(19.0)$ \\
Grade-III & 0 & $(0.0)$ & 0 & $(0.0)$ & 0 & $(0.0)$ & 0 & $(0.0)$ & 0 & $(0.0)$ & 0 & $(0.0)$ \\
\hline Total & 0 & $(0)$ & 18 & $(100)$ & 3 & $(100)$ & 0 & $(0)$ & 0 & $(0)$ & 21 & $(100)$ \\
\hline
\end{tabular}

Table 5: A comparison between known oral pigment studies, where the influence of somker melanin has been evaluated and the present study

\begin{tabular}{llcccc}
\hline Study population & Authors (ref.) & $\mathrm{n}$ & $\begin{array}{l}\text { Melanin } \\
\text { pigmentation }\end{array}$ & $\begin{array}{l}\text { Non- } \\
\text { smoker } \\
\text { melanin } \\
(\%)\end{array}$ & $\begin{array}{c}\text { Smoker } \\
\text { melanin }\end{array}$ \\
& & & $(\%)$ & $(\%)$ \\
\hline Swedish & Hedin 1977(2) & 214 & 18 & 0 & 27 \\
Japanese & Araki et al.1983 (4) & 165 & 18 & 2 & 22 \\
Thai,Malay,Chinese & Hedin\&Axell1991(7) & 243 & 71 & 66 & 18 \\
Indian & Hedin\&Axell1991(7) & 233 & 89 & 85 & 12 \\
Turkish & Unsal et al. 2001 (12) & 496 & 37 & 11 & 27 \\
Bangladeshi & $\begin{array}{l}\text { Yousuf et al. } \\
\text { (present study) }\end{array}$ & 280 & 78 & 8 & 72 \\
& & & & & \\
\hline
\end{tabular}

severe (27 cases) by histological evaluation.

\section{Discussion}

As shown in Table 5, several reports demonstrated that smoker melanosis is found in various populations in Sweden (2,3,5), Japan (4), Indian (7), Thai (7), Malaysia (7) and North America (6). Although these reports indicate that smoking is a critical factor involved in pathogenesis of melanin pigmentation of oral mucosa, they also reveal that the incidence of smoking melanosis differs among ethnic groups examined. Axell and Hedin (3) investigated melanin pigmentation of oral mucosa in a population of 30,118 adult individuals in Sweden and demonstrated that $21.5 \%$ among tobacco smoker were pigmented as compared to $3.0 \%$ among individuals not using tobacco. Unsal et al. (12) demonstrated by studying incidence of oral melanin pigmentation of 496 individuals in a Turkish population that $41.0 \%$ of melanosis occurred in smoker, $13.0 \%$ in former smoker and $46.0 \%$ in non-smoker.
Extensive analysis by Hedin and Axell (7) in Asian populations exhibited higher incidence of melanin pigmentation in smoker $(90.0 \%)$ than that in the European population. In the present study, we found that $82.0 \%$ of the smoker exhibit melanin pigmentation in the Bangladeshi population. This high incidence of melanin pigmentation is in agreement with the previous reports concerning the incidence in Asian populations.

Interestingly, the present study also showed a higher incidence of melanin pigmentation in the nonsmoking Bangladeshi population (58.3\%) than that of the European population (15.0\%). This supports the idea that melanin pigmentation of the oral mucosa might be regulated by genetic background in some ethnic groups.

We found melanin pigmentation in populations of only the $4^{\text {th }}, 5^{\text {th }}$ and $6^{\text {th }}$ decades in our non-smoker group, whereas it was distributed in all decades from the $2^{\text {nd }}$ to $8^{\text {th }}$ in our smoker group. This suggests that melanin pigmentation of oral mucosa in the young generation of 
Bangladeshi population was affected by their smoking habit. Further investigation is needed to confirm this suggestion, in particular since Amir et al. (15) have stated that $13.5 \%$ of Israeli children from 6 to 10 years of age exhibited physiologic melanin pigmentation of oral mucosa.

The present study revealed that gingiva and buccal mucosa were frequently affected sites for melanin pigmentation in both smoker and non-smoker groups in the Bangladeshi population. These results are in agreement with the previous reports describing smoking melanosis in various races. In the smoker group, melanin pigmentation also occurred in the lip, palate and tongue, whereas no pigmentation was observed in these sites in the nonsmoker group. This indicates that smoking incidence of melanin pigmentation at various locations of the oral mucosa other than the gingiva and buccal mucosa.

As shown in Table 4, 16.5\% of melanin pigmentation in the smoker group belonged to Grade III, which is the most intense pigmentation by clinical inspection, whereas no cases of Grade III were found in the nonsmoker group. This suggests that smoking induces not only incidence of melanin pigmentation but also intensity of the pigmentation. Since melanin pigmentation of the oral mucosa increases with consumption of tobacco $(3,4,9,11)$, the consumption may affect intensity of the melanin pigmentation. Unfortunately, the present study lacks details of tobacco consumption of each participant. Such data could possibly support further our assumption.

In conclusion, we demonstrated that a smoking habit affects incidence of melanin pigmentation of oral mucosa in the Bangladeshi population. Incidence of smoking melanosis in Bangladesh is higher than in European populations and it is similar to other Asian populations. These data provide important information for surveying the effects of smoking habits on melanin pigmentation of the oral mucosa among various ethnic groups.

\section{Acknowledgment}

The authors want to express their gratitude to Emeritus Professor Minoru Takagi for his encouragement and advice during this study.

\section{References}

1. Regezi JA, Sciubba JJ and Jordan RCK. Oral Pathology. Clinical pathologic correlations. Saunders, St. Louis. 2003; 131-6.

2. Hedin CA. Smokers' melanosis. Occurrence and localization in the attached gingiva. Arch Dermatol 1977; 113: 1533-8.

3. Axell T and Hedin CA. Epidemiologic study of excessive oral melanin pigmentation with special reference to the influence of tobacco habits. Scand J Dent Res 1982; 90: 434-42.

4. Araki S, Murata K, Ushio K, et al. Dose-response relationship between tobacco consumption and melanin pigmentation in the attached gingiva. Arch Environ Health 1983; 138 375-8.
5. Salonen L, Axell T and Hellden L. Occurrence of oral mucosal lesions, the influence of tobacco habits and an estimate of treatment time in an adult Swedish population. J Oral Pathol Med 1999; 19: 170-6.

6. Brown FH and Houston GD. Smoker's melanosis. A case report. J Periodontol 1991; 62: 524-7.

7. Hedin CA and Axell T. Oral melanin pigmentation in 467 Thai and Malaysian people with special emphasis on smoker's melanosis. J Oral Pathol Med 1991; 20: 8-12.

8. Hedin CA, Pindborg JJ, Daftary DR, et al. Melanin depigmentation of the palatal mucosa in reverse smokers: a preliminary study. J Oral Pathol Med 1992; 21: 440-4.

9. Hedin CA, Pindborg JJ and Axell T. Disappearance of smoker's melanosis after reducing smoking. J Oral Pathol Med 1993; 22: $228-30$

10. Ramer M and Burakoff RP. Smoker's melanosis. Report of a case. NY State J 1997; 63: 20-1.

11. Wallstrom M, Sand L, Nilsson F, et al. The long-term effect of nicotine on the oral mucosa. Addiction 1999; 94: 417-23.

12. Unsal E, Paksoy C, Soykan E, et al. Oral melanin pigmentation related to smoking in a Turkish population. Community Dent Oral Epidemiol 2001; 29: 272-7.

13. Martinez Diaz-Canel AI and Garcia-Pola Vallejo MJ. Epidemiological study of oral mucosal pathology in patients of the Oviedo School of Stomatology. Med Oral 2002; 7: 10-6.

14. Cicek Y and Ertas U. The normal and pathological pigmentation of oral mucous membrane: A review. J Contemp Dental Practice 2003; 3: 1-9.

15. Amir E, Gorsky M, Buchner A, et al. Physiologic pigmentation of the oral mucosa in Israeli children. Oral Surg Oral Med Oral Pathol 1991; 71: 396-8.

(Accepted for publication September 28, 2004) 